\title{
Volumen de contenedores y dosis de fertilizante de liberación controlada en el crecimiento de plantas de Cabralea canjerana producidas en vivero
}

\author{
Container volume and controlled-release fertilizer in growing \\ Cabralea canjerana plants produced in nursery
}

\author{
Suelen Carpenedo Aimi *a, Maristela Machado Araujo b, Enrique Benítez León c, \\ Gisele Gomez de Oliveira ${ }^{\text {b }}$, Fernando da Silva Cunha ${ }^{\text {b }}$ \\ *Autor de correspondência: ${ }^{\text {a }}$ Universidade Federal de Santa Maria, Departamento de Ciências Florestais, \\ Av. Roraima, 1000, CEP 97105-900, Santa Maria, Brasil, tel.: 55-3220-8276, suaimi@gmail.com \\ ${ }^{\mathrm{b}}$ Universidade Federal de Santa Maria, Departamento de Ciências Florestais, Santa Maria, Brasil. \\ ${ }^{\mathrm{c}}$ Universidad Nacional de Asunción, Facultad de Ciencias Agrarias. Campus San Lorenzo, Paraguay.
}

\section{SUMMARY}

The speed at which native tree species are pressured by human action is larger than their conservation established silvicultural knowledge. Cabralea canjerana is a high commercial value species widely distributed throughout Central America and South America; however, its production depends on basic information. The main aim of the current study was to assess the effect of different volume containers as well as that of different controlled-release fertilizer - CRF doses on the production of Cabralea canjerana seedlings, in nursery stage. The following factors were used: volume tubes $\left(110\right.$ and $\left.180 \mathrm{~cm}^{3}\right)$ and doses of CRF $\left(0 ; 2.5 ; 5 ; 7.5 ; 10\right.$ and $12.5 \mathrm{~g} \mathrm{~L}^{-1}$ substrate). The following variables were assessed at 210 days: height, crown diameter, shoot dry matter, root and total, Dickson quality index, leaf area and root length. Higher doses of controlled release fertilizer (FLC) had positive influence on the growth of seedlings. For the production of plants of Cabralea canjerana, the use of containers of $180 \mathrm{~cm}^{3}$ associated with doses of $11.5 \mathrm{~g} \mathrm{~L}^{-1} \mathrm{CRF}-\mathrm{a}$ combination reflected in morphological parameters that can ensure their survival-is recommended in the countryside. The hypothesis that the use of larger volume containers positively affects the growth, independent of the dose of fertilizer used, was accepted. In addition, further studies comparing other combinations of container volume and rates of CRF, different from those used in this work, are recommended, to increase production efficiency in nurseries and guarantee the quality of plants of Cabralea canjerana.

Key words: Meliaceae, forestry nursery, seedling production, base fertilization.

\section{RESUMEN}

La velocidad con que las especies forestales nativas están siendo presionadas por la acción antrópica es mayor que el conocimiento silvicultural establecido para su conversión. Cabralea canjerana es una especie de alto valor comercial, de amplia distribución desde América Central hasta América del Sur, pero aun depende de informaciones básicas para su producción. Así, el objetivo de este estudio fue evaluar el efecto de diferentes volúmenes de contenedores y dosis de fertilizante de liberación controlada-FLC en la producción de mudas de Cabralea canjerana, en la fase de vivero. Los factores utilizados fueron volumen de contenedores $\left(110 \mathrm{y} 180 \mathrm{~cm}^{3}\right) \mathrm{y}$ dosis de FLC $\left(0 ; 2,5 ; 5 ; 7,5 ; 10\right.$ y $12,5 \mathrm{~g} \mathrm{~L}^{-1}$ de sustrato). A los 210 días, fueron evaluadas las variables: altura, diámetro del cuello, masa seca aérea, radical y total, índice de calidad de Dickson, área foliar y largura del sistema radical. Para la producción de plantas de Cabralea canjerana, se recomienda el uso de contenedores de $180 \mathrm{~cm}^{3}$ asociado con dosis de $11,5 \mathrm{~g} \mathrm{~L}^{-1}$ de FLC, combinación reflejada en parámetros morfológicos que pueden asegurar su sobrevivencia en campo. La hipótesis de que la utilización de contenedores de mayor volumen afecta positivamente el crecimiento, independiente de la dosis de fertilización utilizada, fue aceptada. Además, cabe recomendar la realización de estudios que comparen otras combinaciones de volumen de contenedores y dosis de FLC diferentes a las utilizadas en este trabajo, para aumentar la eficiencia de la producción en vivero y garantizar la calidad de plantas de Cabralea canjerana.

Palabras clave: Meliaceae, vivero forestal, producción de plantas, fertilización de base.

\section{INTRODUCCIÓN}

Cabralea canjerana (Vell.) Mart. es una especie perteneciente a la familia Meliaceae, siendo conocida como, canjerana, cedro-canjerana, cancharana, entre otros, presente naturalmente en Costa Rica, Guyana, Perú, Bolivia,
Argentina, Paraguay y Brasil. La especie es plástica, descrita en el grupo sucesional de las pioneras, secundaria tardía o clímax, siendo considerada una de las especies más valiosas, reconocida por su excelente madera y su durabilidad (resistencia a intemperies), además de su valor económico. Aparte de eso, la especie también es indicada para la 
recuperación de áreas alteradas por ser atractiva a la fauna y para el paisajismo (Reitz et al. 1988, Carvalho 2003). Generalmente los valores de altura y diámetro de cuello, pueden ser considerados adecuados para expresar la calidad de las plantas, teniendo en cuenta que Aimi (2014), utilizando plantas de $C$. canjerana con apariencia semejante en plantación (altura $=14,6 \mathrm{~cm}$ y diámetro de cuello $=5,6 \mathrm{~mm}$ ) obtuvo incrementos de $40,88 \mathrm{~cm}$ y $14,59 \mathrm{~mm}$, respectivamente, después de un año, representando un crecimiento de $280 \%$ (altura) y $260 \%$ (diámetro de cuello).

El aumento de la demanda por servicios y productos forestales, con destaque en la producción de plantas de especies nativas, lleva a la necesidad de investigaciones que pueden aportar para aumentar la eficiencia de esa producción, principalmente que sea de bajo costo y de alta calidad, proporcionando elevadas tasas de sobrevivencia y crecimiento de las plantas llevadas al campo.

No obstante, el conocimiento de la producción de plantas y el desarrollo de las diferentes especies en regiones determinadas, es relativamente escaso aun. Tanto para las especies comerciales de rápido crecimiento, así como para las nativas, diversos factores influyen en la producción y calidad de las plantas, entre los cuales es posible citar: la calidad de las semillas, el sustrato utilizado, tipo de contenedor, fertilizantes, irrigación, entre otros.

Con relación a los contenedores, la elección más adecuada depende del tamaño de las semillas, velocidad de crecimiento y el porte deseado de la planta. Desde el punto de vista económico, se debe considerar el costo inicial, disponibilidad de insumos y espacios en el vivero (Landis 1990), siendo que cuando se emplean contenedores mayores existe también el aumento de los costos de producción, debido a la mayor necesidad de espacio, sustrato y otros insumos, además del transporte de las plantas. En ese sentido, las dimensiones de los contenedores para producción de plantas requieren la realización de estudios que contribuyan con informaciones para determinar la mejor relación costo/beneficio, pudiendo considerar la calidad de las plantas producidas como un indicador del beneficio.

Otro aspecto que debe ser llevado en consideración en la producción de plantas en vivero es la fertilización. Una manera de aumentar su eficiencia podría ser el fraccionamiento de los nutrientes, principalmente, nitrógeno y potasio, para evitar pérdidas. Esa práctica, no obstante, resultaría en mayor costo de operaciones, siendo una alternativa la utilización de fertilizantes de liberación controlada (FLC). Conforme a Bustos et al. (2008), la liberación de nutrientes para las plantas, en una sola aplicación, reduciría problemas de toxicidad y pérdidas por lixiviación. Por otra parte, la utilización de FLC exige la realización de estudios sobre las características de estos productos en el establecimiento de las exigencias nutricionales de las plantas en cada etapa de producción (Rose et al. 2004).

En ese contexto, son necesarios estudios técnicos para la producción de plantas de buena calidad, sobre todo para las especies forestales nativas, debido a la demanda para los diversos fines a las que se la pueda utilizar. De esta forma, se plantea la hipótesis de que la utilización de contenedores de mayor volumen afecta positivamente el crecimiento de C. canjerana, independiente de la dosis de fertilización utilizada. En ese sentido, el objetivo de este trabajo es evaluar el desarrollo de plantas de C. canjerana producidas en vivero, en diferentes volúmenes de contenedor y dosis de fertilizante de liberación controlada.

\section{MÉTODOS}

El estudio fue desarrollado en el Laboratorio de Silvicultura y Vivero Forestal del Departamento de Ciencias Forestales (DCFL) $\left(29^{\circ} 43^{\prime} 13^{\prime \prime} \mathrm{S}\right.$ e $\left.53^{\circ} 43^{\prime} 17^{\prime \prime} \mathrm{O}\right)$ de la Universidad Federal de Santa Maria (UFSM), ubicado en el Municipio de Santa Maria, RS, Brasil. De acuerdo a la clasificación de Köppen, el clima de la región es del tipo "Cfa", con lluvias bien distribuidas y precipitación media mensual de 100 a $170 \mathrm{~mm}$ (Alvares et al. 2013).

Los frutos de $C$. canjerana fueron colectados de cinco árboles seleccionados, ubicados en la localidad de Santo Antão, perteneciente al municipio de Santa Maria (RS), en el mes de setiembre de 2011. Posterior a la colecta, los mismos fueron llevados al laboratorio para la extracción de las semillas. La dehiscencia de los frutos fue inducida por medio de su acondicionamiento en envases de polietileno negro, a temperatura ambiente por 12 días. Posteriormente, las semillas fueron extraídas y lavadas con agua corriente para retirada del arilo, luego llevadas a un local sombreado y ventilado por dos días con la finalidad de completar el proceso de secado de las semillas.

El experimento consistió en un esquema factorial $(2 \times 6)$, distribuyéndose los factores entre dos tamaños de tubetes cónicos de polipropileno con capacidad para $110 \mathrm{~cm}^{3}$ (seis estrías, diámetro interno de $35 \mathrm{~mm}$ y altura de $13,5 \mathrm{~cm}$ ) y $180 \mathrm{~cm}^{3}$ (ocho estrías, diámetro interno de $52 \mathrm{~mm}$ y altura de $13 \mathrm{~cm}$ ) y seis dosis de fertilizante de liberación controlada (FLC): 0 (testigo); 2,5; 5,0; 7,5; 10,0 y $12,5 \mathrm{~g} \mathrm{~L}^{-1}$ de sustrato. El experimento fue conducido en diseño experimental completamente aleatorio, con cuatro repeticiones. Los tratamientos, en grupos de dos, fueron distribuidos en bandejas de polipropileno (43,5 x 63,0 x 16,5 cm) con capacidad para 54 celdas, utilizando 24 de ellas para colocar las unidades experimentales correspondiente a cada tratamiento, dejando una hilera central de seis celdas para la separación entre los mismos, totalizando 24 bandejas con 48 unidades de muestreo. De cada unidad de muestreo fueron evaluadas ocho plantas centrales, manteniendo la bordadura, para determinar los diferentes parámetros morfológicos.

El FLC utilizado fue Osmocote ${ }^{\circledR}$, cuya dosis recomendada por el fabricante para especies nativas es de 5 a $8 \mathrm{~g}$ $\mathrm{L}^{-1}$ de sustrato con la siguiente composición química: macronutrientes $15 \%$ de nitrógeno $(\mathrm{N}) ; 9,0 \%$ de superfosfato $\left(\mathrm{P}_{2} \mathrm{O}_{5}\right)$ y $12 \%$ de clorato de potasio $\left(\mathrm{K}_{2} \mathrm{O}\right) ; 1,0 \%$ de magnesio $(\mathrm{Mg})$ y los micronutrientes $2,3 \%$ de azufre (S); $0,05 \%$ 
de cobre $(\mathrm{Cu}) ; 0,06 \%$ de manganeso (Mn), $0,45 \%$ de hierro $(\mathrm{Fe})$ e $0,2 \%$ de molibdeno (Mo). Según las indicaciones del fabricante, en sustrato húmedo (temperatura media de $21{ }^{\circ} \mathrm{C}$ ), la liberación de los nutrientes ocurre en seis meses.

Los tubetes fueron llenados con sustrato comercial compuesto por turfa de Sphagnum sp., vermiculita expandida y cascarilla de arroz carbonizada. Las características del producto, conforme informaciones del fabricante son: pH 5,0 ( $\pm 0,5)$; conductividad eléctrica (CE) $0,7 \mathrm{mS} \mathrm{cm}^{-1}$ $( \pm 0,3)$; densidad $114 \mathrm{~kg} \mathrm{~m}^{-3} \mathrm{y}$ capacidad de retención de agua (CRA) $55 \%$. El sustrato fue humedecido y, al mismo tiempo, fue mezclado el fertilizante de base (FLC), con la dosis correspondiente a cada tratamiento.

Posterior a la siembra, las bandejas fueron trasladadas para invernáculos donde permanecieron hasta los 210 días. El riego fue realizado por una barra de irrigación con microaspersores, con un caudal de $4 \mathrm{~mm} \mathrm{dia}^{-1}$, accionada cuatro veces por día, por un temporizador.

Además de la fertilización de base, con FLC, fue realizada una fertilización de cobertura a los 150 días en todos los tratamientos, con fertilizante conteniendo: $9 \%$ de N; $45 \%$ de $\mathrm{P}_{2} \mathrm{O}_{5} ; 15 \%$ de $\mathrm{K}_{2} \mathrm{O}$ y $1 \%$ de magnesio $(\mathrm{Mg})$, conforme informaciones del embalaje del producto. La dosis utilizada fue de $3 \mathrm{~g} \mathrm{~L}^{-1}$, disuelto y aplicado vía solución acuosa con pulverizador costal, semanalmente, hasta el final del experimento.

Las evaluaciones de los parámetros morfológicos fueron realizadas a los 210 días después de la emergencia, midiéndose altura de la planta $(\mathrm{cm})$, con una regla milimetrada y diámetro en la base del cuello ( $\mathrm{mm})$, con un calibre digital. La cuantificación de la masa seca de la parte aérea y radical de las plantas fue realizada, basándose en la muestra de cuatro plantas por tratamiento. Las raíces fueron lavadas con agua corriente con el auxilio de un tamiz (malla de $0,84 \mathrm{~mm}$ ), colocadas en frascos de vidrio con $100 \mathrm{~mL}$ de solución de etanol (12\%) y almacenadas en cámara fría (temperatura de $8-10{ }^{\circ} \mathrm{C}$ y $90 \%$ de humedad relativa), hasta el momento de las evaluaciones de la longitud del sistema radical.

Para la determinación del área foliar (AF) y la longitud del sistema radical (LR) fueron utilizadas las mismas muestras para la obtención de masa seca, siendo pesadas antes del secado del material. La determinación del área foliar y la longitud del sistema radical fue realizada de la siguiente manera: las hojas y las raíces de las plantas de C. canjerana fueron distribuidas sobre un papel blanco, tamaño A4 (con escala), posteriormente fue prensada por un vidrio transparente y fotografiada con una cámara digital SONY (modelo DSC-T100), apoyada en una estructura fija de $0,18 \mathrm{~m}$ y zoom de 1,4x. Las imágenes fueron editadas con el uso de aplicativos IrfanView y, posteriormente, procesadas con el software para análisis de imágenes UTHSCSA $\AA$ ImageTool for Windows versión 3.00@ , obteniéndose el área foliar y la longitud del sistema radical.

Posterior a la obtención de las imágenes, las muestras fueron colocadas en embalajes de papel, identificadas y lle- vadas a una estufa con circulación de aire forzado a $65^{\circ} \mathrm{C}$, hasta obtener peso constante, y pesadas en una balanza analítica (precisión de $0,001 \mathrm{~g}$ ) para determinar la masa seca aérea y la masa seca radical, obteniéndose de esta manera la masa seca total y el índice de calidad de Dickson (Dickson et al. 1960), este último índice fue calculado utilizando la fórmula:

$$
\mathrm{ICD}=\mathrm{MST} /(\mathrm{H} / \mathrm{DC}+\mathrm{MSA} / \mathrm{MSR}) \quad[\mathrm{g}, \mathrm{cm} / \mathrm{mm}, \mathrm{g} / \mathrm{g}][1]
$$

Donde: $\mathrm{ICD}=$ índice de calidad de Dickson, $\mathrm{MST}=$ masa seca total, $\mathrm{H}=$ altura de la parte aérea, $\mathrm{DC}=$ diámetro del cuello, $\mathrm{MSA}=$ masa seca aérea y $\mathrm{MSR}=$ masa seca radical.

Fueron verificados los supuestos de normalidad y homogeneidad y, posteriormente, los datos fueron sometidos a análisis de varianza. Cuando constatada la existencia de diferencia significativa entre los tratamientos por medio de la prueba de F, se realizó la comparación de medias por la prueba t, prueba Scott-Knott o regresión polinomial. Para realizar los análisis fue utilizado el software Sisvar (Ferreira 2011). Además, fue realizada la correlación de Pearson (r) $(P<0,05)$ con el suplemento Excel Action.

El cálculo de la máxima eficiencia técnica (DMET) fue realizado de acuerdo con la metodología descrita en Storck et al. (2000), por medio de la siguiente fórmula:

$$
\mathrm{X}=-\mathrm{b}_{1} / 2 \mathrm{~b}_{2}
$$

Donde: $\mathrm{X}=$ punto da máxima eficiencia técnica; $\mathrm{b}_{1}$ y $\mathrm{b}_{2}=$ coeficientes de la ecuación.

\section{RESULTADOS}

Fue verificada interacción significativa entre los tratamientos 210 días después de la siembra (DDS) para las variables: altura $(P=0,001)$ y diámetro de cuello $(P=0,004)$, y efecto significativo de los factores independientes para las variables: masa seca aérea $(P=0,001)$, masa seca radical $(P=0,001)$, masa seca total $(P=0,001)$, índice de calidad de Dickson $(P=0,001)$, área foliar $(P=0,001)$ y longitud del sistema radical $(P=0,02)$.

El crecimiento en altura $(\mathrm{H})$ presentó comportamiento cuadrático con dosis de máxima eficiencia técnica (DMET) estimada en $9,2 \mathrm{~g} \mathrm{~L}^{-1}$ para el tubete de $110 \mathrm{~cm}^{3}$ $(\mathrm{H}=12,49 \mathrm{~cm})$ y $11,5 \mathrm{~g} \mathrm{~L}^{-1}$ para el tubete de $180 \mathrm{~cm}^{3}$ $(\mathrm{H}=14,11 \mathrm{~cm})$ (figura 1A). La variable diámetro del cuello (DC) también presentó comportamiento cuadrático (figura 1B), la dosis de máxima eficiencia técnica fue de $9,1 \mathrm{~g} \mathrm{~L}^{-1}$ para el contenedor de $110 \mathrm{~cm}^{3}(4,47 \mathrm{~mm})$ y $8,6 \mathrm{~g}$ $\mathrm{L}^{-1}$ para tubete de $180 \mathrm{~cm}^{3}(4,67 \mathrm{~mm})$.

El aumento de las dosis de FLC proporcionó mayor masa seca aérea, masa seca radical y masa seca total (figura 2A), índice de calidad de Dickson (figura 2B), área foliar y longitud del sistema radical (figura 3A y B), demostrando que esas variables tuvieron crecimiento con 

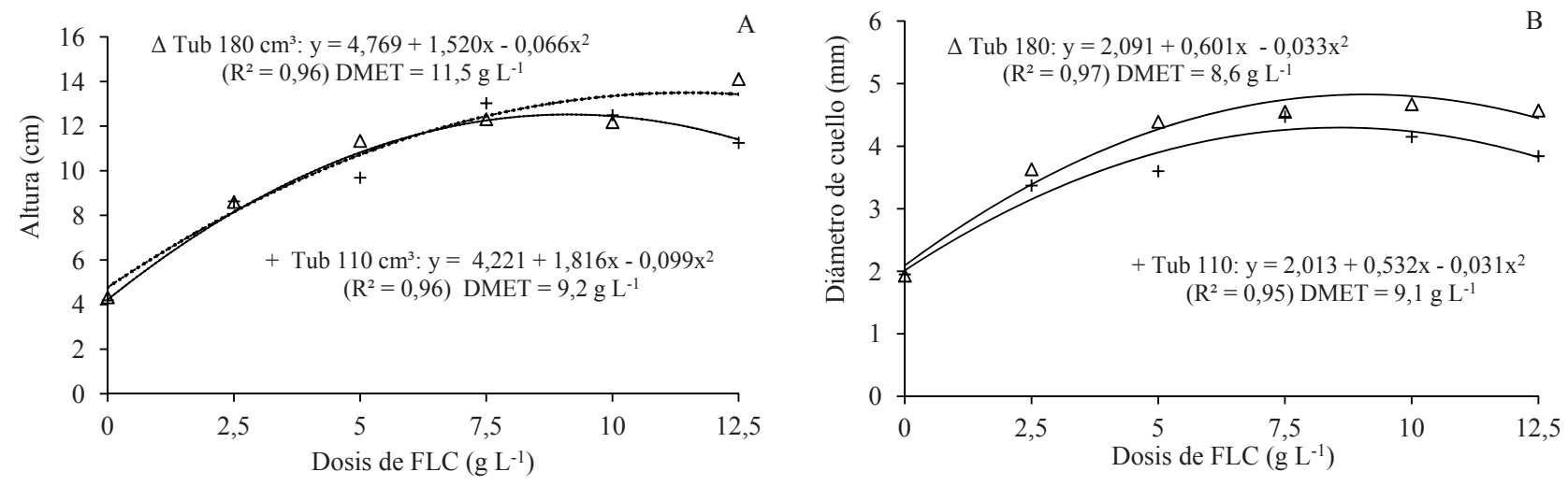

Figura 1. Crecimiento en altura (A) y diámetro del cuello (B) de plantas de C. canjerana, en diferentes tamaños de tubetes y dosis de fertilizante de liberación controlada (FLC), a los 210 días después de la siembra en la fase de vivero.

Height growth (A) and root collar diameter (B) of Cabralea canjerana seedlings in different size tubes and different controlled-release fertilizer doses at 210 days after sowing, in the nursery stage.
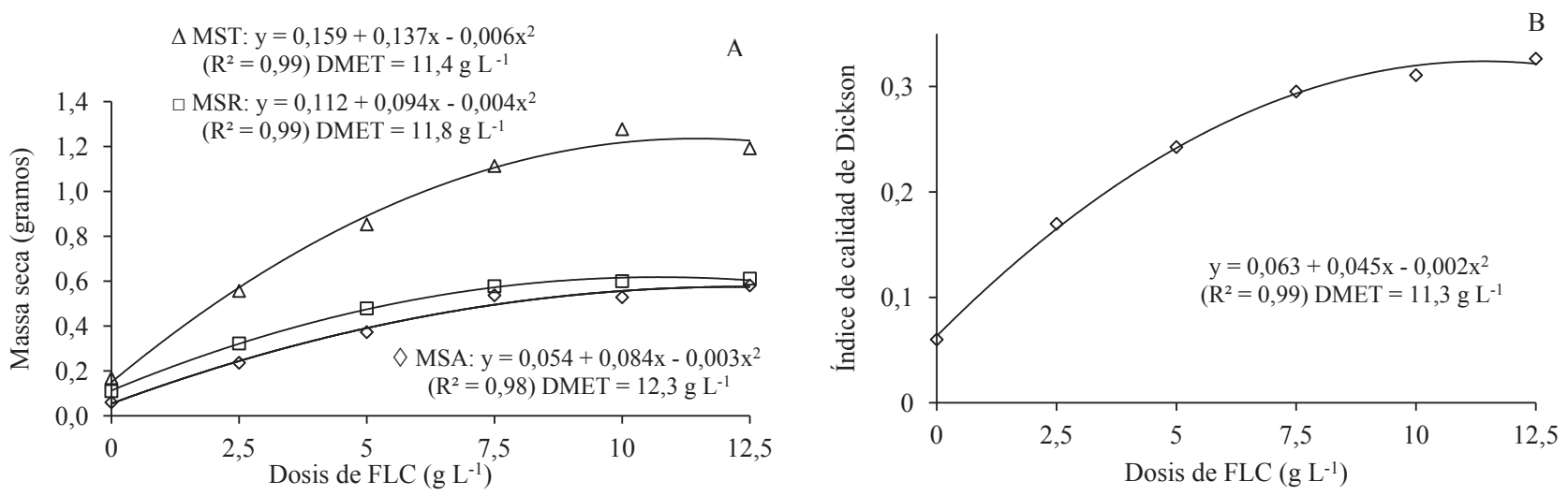

Figura 2. Comportamiento de masa seca aérea (MSA), masa seca radical (MSR), masa seca total (MST) (A) e Índice de calidad de Dickson (B) de plantas de C. canjerana a los 210 días, en función de las dosis de fertilizante de liberación controlada (FLC) en la fase de vivero.

Behavior of shoot dry matter (MSA), root dry matter (MSR), total dry matter (MST) (A) and Dickson quality index (B) of Cabralea canjerana seedlings evaluated at 210 days, based on the doses of controlled-release fertilizer (FLC), during the nursery stage.
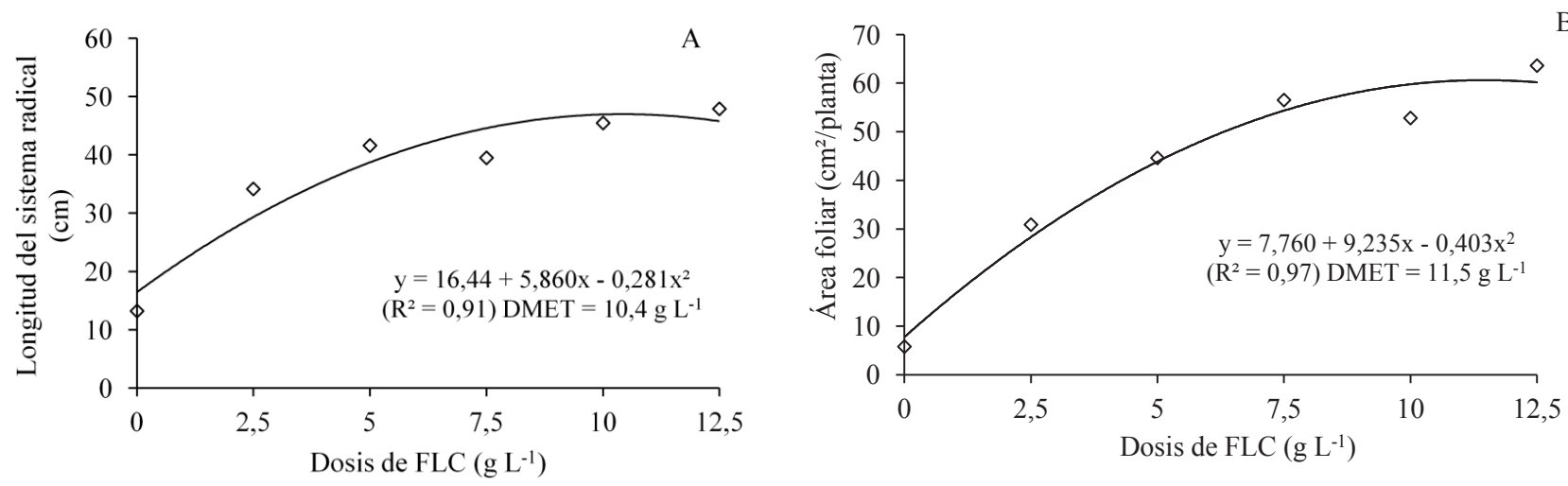

Figura 3. Longitud radical (A) y comportamiento del área foliar (B) de plantas de C. canjerana, evaluadas a los 210 días, en función de las dosis de fertilizante de liberación controlada, en la fase de vivero.

Root length (A) and leaf area (B) behavior of Cabralea canjerana seedlings evaluated at 210 days, depending on controlled-release fertilizer doses in the nursery. 
comportamiento cuadrático, cuya dosis de máxima eficiencia técnica fue igual o superior a 10,4 g de fertilizante de liberación controlada por litro de sustrato.

De forma semejante, el contenedor de $180 \mathrm{~cm}^{3}$ permitió mayor crecimiento de masa seca aérea (41\%), masa seca total (32\%), índice de calidad de Dickson (29\%), área foliar $(26 \%)$ y longitud del sistema radical (19\%) (cuadro 1). Esas respuestas corresponden a aquellas verificadas por medio de las variables altura $(P=0,001)$ y diámetro de cuello $(P=0,004)$, demostrando que la especie tiene potencial para un crecimiento más satisfactorio con mayor volumen de sustrato y dosis de fertilizante.

Con relación a masa seca aérea y masa seca radical (figura 2A) con la dosis por encima de $10 \mathrm{~g} \mathrm{~L}^{-1}$ las medias de esas variables quedaron prácticamente iguales. Teniendo en cuenta que las variables están directamente correlacionadas (cuadro 2), se observó que el mayor crecimiento en altura proporcionó ganancia en biomasa aérea y, consecuentemente, en masa seca aérea.

\section{DISCUSIÓN}

Se observa que a los 210 días, la mayor altura de las plantas es para el tubete de $180 \mathrm{~cm}^{3}(\mathrm{H}=14,11 \mathrm{~cm})$ con $12,5 \mathrm{~g}$ de fertilizante de liberación controlada por litro de sustrato, mientras que en el tubete de $110 \mathrm{~cm}^{3}$ el promedio para esa variable es $11,25 \mathrm{~cm}$, lo que puede llevar a inferir que este recipiente no promueve un buen crecimiento en altura de las plantas de C. canjerana, atendiendo que la dosis de fertilizante de liberación controlada utilizada es la misma en los dos recipientes, pero en el de $180 \mathrm{~cm}^{3}$ existe mayor espacio interno para el desarrollo del sistema radical. Por otra parte, se observan síntomas de clorosis en las plantas, a los tres meses después de la emergencia, considerando la coloración amarilla (5Y 7/10) de las hojas, determinada mediante Munsell (1977), sugiere toxicidad.

Para el diámetro del cuello, independiente del contenedor y, sin considerar el testigo, a los 210 días en vivero, todas las plantas de C. canjerana presentan diámetro de cuello

Cuadro 1. Medias de los parámetros morfológicos masa seca aérea (MSA), masa seca radical (MSR), masa seca total (MST), índice de calidad de Dickson (ICD), área foliar (AF) y longitud del sistema radical (LR) de plantas de C. canjerana, a los 210 días en vivero, producidas en contenedores de 110 y $180 \mathrm{~cm}^{3}$.

Morphological parameters average of shoot dry matter (MSA), root dry matter (MSR), total dry matter (MST), Dickson quality index (ICD), leaf area (LR) and Root length (AF) of Cabralea canjerana seedlings, at 210 days in the nursery, produced in containers of 110 and $180 \mathrm{~cm}^{3}$.

\begin{tabular}{ccccccc}
\hline Tubete $\left(\mathrm{cm}^{3}\right)$ & MSA $(\mathrm{g})$ & MSR $(\mathrm{g})$ & MST $(\mathrm{g})$ & ICD & AF $\left(\mathrm{cm}^{2}\right)$ & LR $(\mathrm{cm})$ \\
\hline 110 & $0,2987 \mathrm{~b}^{*}$ & $0,392 \mathrm{~ns}^{* *}$ & $0,6894 \mathrm{~b}$ & $0,1937 \mathrm{~b}$ & $37,3242 \mathrm{~b}$ & $33,0256 \mathrm{~b}$ \\
180 & $0,5064 \mathrm{a}$ & 0,5079 & $1,0139 \mathrm{a}$ & $0,2746 \mathrm{a}$ & $50,7650 \mathrm{a}$ & $40,8958 \mathrm{a}$ \\
\hline
\end{tabular}

*Medias seguidas de la misma letra, en la columna, no difieren entre sí por la prueba t de Student al $5 \%$ de probabilidad de error; **ns: no significativo por la prueba $\mathrm{F}$.

Cuadro 2. Matriz de correlación de Pearson (r) de los parámetros morfológicos de las plantas de C. canjerana producidas a los 210 días en vivero.

Pearson's correlation matrix (r) of morphological parameters of Cabralea cajerana seedlings produced at 210 days in the nursery.

\begin{tabular}{ccccccccc}
\hline Variabel & $\mathrm{H}$ & $\mathrm{DC}$ & MSA & MSR & MST & ICD & AF & LR \\
\hline H & 1 & & & & & & & \\
DC & $0,98^{*}$ & 1 & & & & & \\
MSA & $0,92^{*}$ & $0,91^{*}$ & 1 & & & & \\
MSR & $0,89^{*}$ & $0,91^{*}$ & $0,94^{*}$ & 1 & & & \\
MST & $0,92^{*}$ & $0,93^{*}$ & $0,98^{*}$ & $0,98^{*}$ & 1 & & \\
ICD & $0,89^{*}$ & $0,92^{*}$ & $0,95^{*}$ & $0,99^{*}$ & $0,99^{*}$ & 1 & & \\
AF & $0,93^{*}$ & $0,92^{*}$ & $0,98^{*}$ & $0,90^{*}$ & $0,96^{*}$ & $0,92^{*}$ & 1 & \\
LR & $0,91^{*}$ & $0,93^{*}$ & $0,91^{*}$ & $0,91^{*}$ & $0,93^{*}$ & $0,91^{*}$ & $0,91^{*}$ & 1 \\
\hline
\end{tabular}

*significativo a $P<0,05 ; \mathrm{H}=$ altura; $\mathrm{DC}=$ diámetro del cuello; $\mathrm{MSA}=$ masa seca aérea; $\mathrm{MSR}=$ masa seca radical; $\mathrm{MST}=$ masa seca total; ICD = índice de calidad de Dickson; $\mathrm{AF}$ = área foliar y $\mathrm{LR}=$ longitud radical. 
superior a $3 \mathrm{~mm}$, siendo este valor considerado como patrón de calidad en especies forestales (Gomes y Paiva 2011).

Aparte de las condiciones de producción, referente a los factores estudiados en el presente trabajo, el periodo de permanencia de las plantas en el vivero también puede ejercer influencia sobre la calidad de las mismas. En ese sentido, Gasparin et al. (2014), en plantas de C. canjerana producidas en diferentes composiciones de sustratos, asociados al volumen de contenedores de 100 y $280 \mathrm{~cm}^{3}$, a los 330 días de permanencia en el vivero, obtuvieron altura de 16,2 y $16,5 \mathrm{~cm}$ y diámetro del cuello de 7,11 y 6,84 $\mathrm{mm}$, respectivamente, valores relativamente mayores a la media obtenida en este estudio. No obstante, es importante considerar que el tiempo de permanencia en vivero entre los estudios comparados es diferente.

En especies de rápido crecimiento, como Eucalyptus saligna Sm. y Eucalyptus grandis W. Hill., que son especies comúnmente utilizadas en reforestaciones, cuando presentan valores alrededor de 3,5 $\mathrm{mm}$ de $\mathrm{DC}$, redundan en elevada sobrevivencia y crecimiento posterior al transplante (Silveira et al. 2004). De esa forma, se observa que C. canjerana presenta expresiva acumulación de carbono en el crecimiento del diámetro en relación a la altura, características importantes que conforme Ritchie et al. (2010) condiciona la mayor sobrevivencia de especies arbóreas, principalmente en locales de estrés para la planta.

La ganancia de masa seca, aérea y radical, de las plantas está directamente relacionada con el tamaño del contenedor utilizado. Al igual que los resultados obtenidos en este estudio, Brachtvogel y Malavasi (2010), obtuvieron la misma relación en plantas de Peltophorum dubium Taub.

De igual forma, el aumento del volumen de sustrato utilizado para producir plantas en vivero permite el incremento en masa seca radical, posibilitando aumentar la circulación y la absorción de nutrientes y, consecuentemente, el crecimiento de la parte aérea (Serrano et al. 2006). Conforme Marenco y Lopes (2007), la parte aérea de la planta puede ser un reservorio temporario de asimilados, que cuando sea necesario son translocados y utilizados para la formación de hojas, responsables por la captación de energía solar.

El área foliar y la longitud del sistema radical también incrementan en el contenedor de $180 \mathrm{~cm}^{3}$ con las dosis más elevadas de fertilizante, lo que puede ser considerado favorable para el desarrollo de la planta después del transplante en su lugar definitivo (cuadro 1). Según Kozlowski et al. (1991), elevada área foliar corresponde a mayor producción de fotoasimilados debido al aumento de la captación de luz, en esa condición, las mudas llevadas al campo presentan crecimiento inicial más rápido.

Otra variable considerada importante para la evaluación de la calidad de plantas es el índice de calidad de Dickson, siendo mencionado como un parámetro completo. No existe en la literatura valores de referencia en cuanto a calidad para especies arbóreas nativas, lo que dificulta el análisis de los índices obtenidos, a pesar de ser claro que mayor índice de calidad de Dickson representa plantas de mejor calidad. De manera general, Birchler et al. (1998) recomiendan que ese índice debe ser $>0,2$ para que la planta presente buena calidad y elevada tasa de crecimiento y sobrevivencia después del transplante al campo. Así, el índice de calidad de Dickson $(0,3)$ obtenido con la dosis de máxima eficiencia técnica 10,3 $\mathrm{g} \mathrm{L}^{-1}$ (figura $3 \mathrm{~B}$ ) puede ser considerado un valor de referencia para plantas de $C$. canjerana.

No obstante, las variables utilizadas para evaluar el crecimiento de las plantas están bien relacionadas (cuadro 2). La mayoría de las variables se encuentran fuertemente correlacionadas entre sí (Callegari-Jacques 2003). Para la longitud radical se observa correlación positiva y significativa con todos los demás parámetros, siendo que los resultados más expresivos son verificados con el diámetro de cuello. Además, considerando el trabajo que implica obtener la longitud de la raíz, los valores de diámetro de cuello pueden expresar el desarrollo del sistema radical de las plantas de $C$. canjerana en vivero, ya que existe una correlación positiva entre ambas variables.

La hipótesis propuesta: la utilización de contenedores de mayor volumen afecta positivamente el crecimiento de C. canjerana, independiente de la dosis de fertilización utilizada, es aceptada. Realizando una sumatoria de los resultados, puede observarse que para la mayoría de las variables (altura, diámetro de cuello, masa seca aérea, masa seca radical, masa seca total, índice de calidad de Dickson, área foliar y longitud del sistema radical), el contenedor con mayor volumen de sustrato $\left(180 \mathrm{~cm}^{3}\right)$ posibilita mayor crecimiento de las plantas y mayor tiempo de permanencia en vivero sin perjudicar el sistema radical. La masa seca radical fue indiferente, por otra parte, ninguna variable expresa calidad superior de la planta cuando son producidas en el tubete de $110 \mathrm{~cm}^{3}$, lo cual puede llevar a inferir que resulta restrictivo para el desarrollo de la especie. La dosis de fertilizante de liberación controlada varia de $8,6 \mathrm{~g} \mathrm{~L}^{-1}$ a $12,3 \mathrm{~g} \mathrm{~L}^{-1}$, no obstante, para las variables altura, masa seca total, índice de calidad de Dickson y área foliar, la dosis de máxima eficiencia técnica es alrededor de $11,5 \mathrm{~g} \mathrm{~L}^{-1}$. Esa dosis puede ser considerada alta, atendiendo el costo del producto, no obstante, su utilización puede prescindir del empleo de mano de obra con fertilización de cobertura durante algunas fases de la producción en vivero. Otro hecho que debe ser destacado es que $C$. canjerana, a pesar de ser una especie con crecimiento lento en altura, en el vivero, utiliza dosis elevadas de fertilizante de liberación controlada para agregar en área foliar y biomasa de base (tallo y raíz), factores que pueden ser considerados determinantes en el momento del establecimiento de las plantas en campo.

\section{CONCLUSIONES}

La hipótesis propuesta: la utilización de contenedores de mayor volumen afecta positivamente el crecimiento de Cabralea canjerana, independiente de la dosis de fertilización utilizada, es aceptada. 
Para la producción de plantas de C. canjerana en vivero, se recomienda el uso de contenedores de polipropileno de $180 \mathrm{~cm}^{3}$ asociado con dosis de $11,5 \mathrm{~g} \mathrm{~L}^{-1}$ de fertilizante de liberación controlada, combinación reflejada en parámetros morfológicos que pueden asegurar su sobrevivencia en campo.

Además, cabe recomendar la realización de estudios que comparen otras combinaciones de volumen de contenedores y dosis de fertilizante de liberación controlada diferentes a las utilizadas en este trabajo, para aumentar la eficiencia de la producción en vivero y garantizar la calidad de plantas de C. canjerana.

\section{AGRADECIMIENTOS}

A la Coordenação de Aperfeiçoamento de Pessoal de Nível Superior (CAPES) por el apoyo para la beca de maestría de la primera autora, al Conselho Nacional de Desenvolvimento Científico e Tecnológico (CNPq) por la beca de productividad científica de la segunda autora. $\mathrm{Al}$ señor Élio Campanhol por la ayuda brindada en la ejecución del experimento.

\section{REFERENCIAS}

Aimi SC. 2014. Tecnologia de sementes e crescimento inicial de mudas de Cabralea canjerana (Vell.) Mart. Master Thesis (Mestrado em Engenharia Florestal). Santa Maria, Brasil. Universidade Federal de Santa Maria. 110 p.

Alvares CA, JL Stape, PC Sentelhas, JLM Gonçalves, G Sparovek. 2013. Köppen's climate classification map for Brazil. Meteorologische Zeitschrift 22: 711-728.

Birchler T, RW Rose, A Royo, M Pardos. 1998. La planta ideal: revisión del concepto, parámetros definitorios e implementación práctica. Investigacion Agraria, Sistemas y Recursos Forestales (Madrid) 7(1/2): 109-121.

Bustos F, ME González, P Donoso, V Gerding, C Donoso, B Escobar. 2008. Efectos de distintas dosis de fertilizante de liberación controlada (Osmocote $($ ) en el desarrollo de plantas de coigüe, raulí y ulmo. Bosque 29(2): 155-161.

Brachtvogel EL, UC Malavasi. 2010. Volume do recipiente, adubação e sua forma de mistura ao substrato no crescimento inicial de Peltophorum dubium (Sprengel) Taubert em viveiro. Revista Árvore 34(2): 223-232.

Carvalho PER. 2003. Espécies Arbóreas Brasileiras. Brasília: EMBRAPA Informação Tecnológica. Colombo, Brasil. EMBRAPA Florestas. 1039 p.
Callegari-Jacques SM. 2003. Bioestatística: princípios e aplicações. Porto Alegre, Brazil. Artmed. 255 p.

Dickson A, AL Leaf, JF Hosner. 1960. Quality appraisal of while spruce and white pine seedling stock in nurseries. Forestry Chronicle 36:11-13.

Ferreira DF. 2011. Sisvar: a computer statistical analysis system. Ciência e Agrotecnologia 35(6): 1039-1042.

Gasparin E, AL de Avila, MM Araujo, AF Cargnelutti, DU Dorneles, DRB. 2014. Influência do substrato e do volume de recipiente na qualidade das mudas de Cabralea canjera$n a$ (Vell.) Mart. em viveiro e no campo. Ciência Florestal 24(3): 553-563.

Gomes JM, HN Paiva. 2011. Viveiros florestais (Propagação sexuada). Cadernos didáticos 72. Viçosa, Brasil. UFV. 116 p.

Kozlowski, TT, PJ Kramer, SG Pallardy. 1991. The physiological ecology of woody plants. New York, U.S.A. Academic Press. $657 \mathrm{p}$.

Landis TD. Containers: types and functions. 1990. In Landis TD, RK Dumroese, DL Haase eds. The container tree nursery manual. Agriculture Handbook 674. Washington DC, U.S.A. Department of Agriculture, Forest Service. p. 1-40.

Marenco RA, NF Lopes. 2007. Fisiologia vegetal: fotossíntese, respiração, relações hídricas e nutrição mineral. Viçosa, Brasil. Ed. da UFV. 469 p.

Munsell Color Charts. 1977. Munsell color charts for plant tissues. Gretagmacbeth 617, Little Britain Road, New Windsor, New York 12553-6148. 20 p.

Reitz R, RM Klein, A Reis. 1988. Projeto madeira do Rio Grande do Sul. Porto Alegre, Brazil. Secretaria da Agricultura e Abastecimento. 525 p.

Ritchie GA, TD Landis, RK Dumroese, DL Haase. 2010. Assessing plant quality. Seedling Processing, Storage, and Outplanting. Vol. 7, Agriculture Handbook 674. Washington DC, U.S.A. Department of Agriculture Forest Service. 200 p.

Rose R, DL Haase, E Arellano. 2004. Fertilizantes de entrega controlada: potencial para mejorar la productividad de la reforestación. Bosque 25(2): 89-100.

Serrano LAL, CMM da Silva, J Ogliari, AJC del Carvalho, CS Marinho, E Detmann. 2006. Utilização de substrato composto por resíduos da agroindústria canavieira para produção de mudas de maracujazeiro-amarelo. Revista Brasileira de Fruticultura 28(3): 487-491.

Stork L, DC Garcia, SJ Lopes. 2000. Experimentação vegetal. Santa Maria: Ed. UFSM, 198p.

Silveira, RLV de A, A Moreira, EN Higashi. 2004. Crescimento e sobrevivência de mudas de eucalipto sob doses de boro cultivadas em condições de viveiro e de campo. Ciência e Agrotecnología 28(2): 366-371. 
